\title{
Is there sufficient evidence to justify changes in dietary habits in heart failure patients? A systematic review
}

\author{
Alejandra García-García ${ }^{1}$, Luis A. Alvarez-Sala-Walther ${ }^{1}$, Hae-Young Lee ${ }^{2}$, Cristina Sierra ${ }^{3}$, \\ Domingo Pascual-Figal ${ }^{4}$, and Miguel Camafort ${ }^{3}$
}

\begin{abstract}
${ }^{1}$ Department of Internal Medicine, "Gregorio Marañon" University General Hospital, Madrid, Spain; ${ }^{2}$ Department of Internal Medicine, Seoul National University Hospital, Seoul, Korea; ${ }^{3}$ Department of Internal Medicine-ICMiD, Hospital Clinic, University of Barcelona, Barcelona; ${ }^{4}$ Department of Cardiology, "Virgen de la Arrixaca" University General Hospital, University of Murcia, Murcia, Spain
\end{abstract}

Received : November 24, 2020 Accepted: February 8, 2021

\section{Correspondence to}

Miguel Camafort, M.D.

Department of Internal Medicine-

ICMiD, Hospital Clinic, Universidad

de Barcelona, C/. Villarroel 170,

E-08036 Barcelona, Spain

Tel: +34-932275000

Fax: +34-932275759

E-mail: camafort@clinic.cat

https://orcid.org/0000-0002-8669-

6410
The incidence and prevalence of heart failure (HF) is increasing worldwide, leading to high morbidity and mortality. The global management of HF involves lifestyle changes in addition to pharmacological treatments. Changes include exercise and dietary recommendations, mainly salt and fluid restriction, but without any clear evidence. We conducted a systematic review to analyse the degree of evidence for these dietary recommendations in HF. Only randomized controlled trials (RCT), and observational studies in humans were selected. Studies were considered eligible if they included participants with HF and sodium and/or fluid restriction. Publications in languages other than English or Spanish were excluded. We included 15 studies related to sodium or fluid restriction. Nine RCT and six observational studies showed some improvements in symptoms and quality of life and a degree of reduction in new hospitalizations, but the results are based on limited population groups, applying different methodologies, and with different restriction goals. We found a lack of clear evidence of the benefits of sodium/fluid restriction in chronic HF. The evidence is limited to few studies with conflicting results. Randomized clinical trials are needed to fill this gap in our knowledge.

Keywords: Heart failure; Diet, sodium-restricted; Diet therapy

\section{INTRODUCTION}

Heart failure (HF) is a complex syndrome whose incidence and prevalence is growing worldwide, implying high morbidity and mortality. The global management of HF involves pharmacological and non-pharmacological treatments. Pharmacological treatments have improved greatly in recent years $[1,2]$ but non-pharmacological treatments continue to be based on recommendations for lifestyle changes that have not changed over recent years [3]. In the 2016 Guidelines of the European Society of Cardiology, they are limited to a sub-index, specifying avoidance of excessive fluid intake (1.5 to $2 \mathrm{~L}$ /day) with a grade of recommendation of II-a, and a level of evidence of $C$. Sodium restriction is recommended in symptomatic HF to avoid excessive sodium intake (> 6 $\mathrm{g} /$ day) (Grade of recommendation I, level of evidence C). [4]. However, the potential benefits of lifestyle modifications have not been fully proven by randomized controlled trials 
(RCTs) or cohort studies [5].

However, these recommendations are based on Western dietary and cultural traditions, although other cultures, such as those of Asia, generally have a higher salt intake than Westerners. Recent studies indicate that salt consumption remains high throughout the Asia-Pacific region [6-9], including countries such as Australia (9.6 g/day), China (9.1 g/ day), Korea (10 g/day), Japan (11 g/day), and India (9.45 to $10.41 \mathrm{~g} /$ day). These estimated intakes are almost double the World Health Organization's recommended maximum daily salt intake of $5 \mathrm{~g} /$ day, mainly due to the over-consumption of spices, vegetables, and grains $[10,11]$. A recent study of pooled data from European, American, and Asian populations with $\mathrm{HF}$ (with reduced ejection fraction) showed that the rate of cardiovascular death/hospitalization for HF is higher in Asia than in Western Europe and North America [12]. This has also been shown in Korean HF registries [13].

Current recommendations are mainly based on available evidence from related cardiovascular diseases, which has had a large impact in reducing events $[14,15]$, with no differences in guidelines between regions [16-19].

It has been assumed that such changes in dietary patterns have a favourable impact on the prognosis of HF. However, the results are unclear with respect to sodium and fluid restriction [20]. Therefore, we carried out a systematic review to analyse the available evidence of salt and fluid restriction on outcomes in HF patients.

\section{METHODS}

We made a systematic search in Medline and the Cochrane Central Register of Controlled Trials using the search strategy shown in Fig. 1. Only RCT and observational studies in humans were selected. Studies were considered eligible if they included participants with HF and sodium or fluid restriction. Publications in languages other than English or Spanish were excluded. The search was conducted on September 1, 2019.

After eliminating duplicates and non-relevant articles (no adequate endpoints, such as rehospitalization or mortality, or unclear or inadequate methodologies), the articles were analysed independently by A.G.G. and M.C. The final list of studies included was decided by discussion between the authors and full agreement was required. The study selection flowchart is shown in Fig. 1. Finally, we included 15 studies

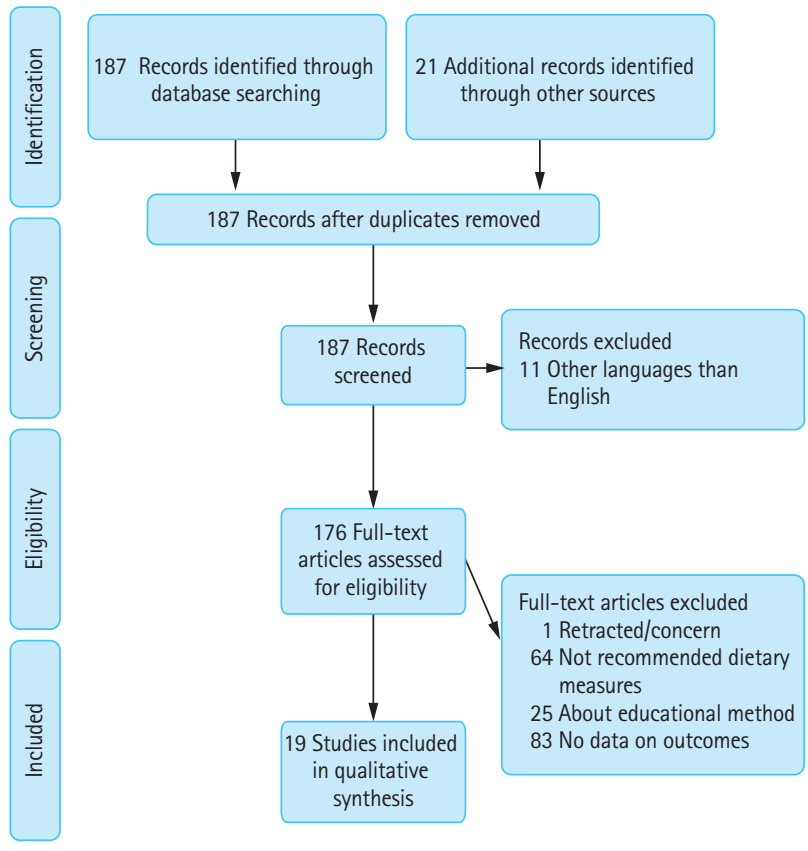

Figure 1. Preferred Reporting Items for Systematic reviews and Meta-Analyses (PRISMA) flow diagram search strategy (/(("heart failure"[Title/Abstract] OR (/(()(((“preservation, biological"[MeSH Terms] OR ("preservation"[All Fields] AND "biological"[All Fields])) OR "biological preservation"[All Fields]) OR "preservation."

related to sodium or fluid restriction.

\section{RESULTS}

Restriction of daily sodium intake, recommended for HF patients, has been assessed in different studies in order to confirm the benefits of a low sodium diet on HF evolution.

\section{Randomized controlled trials}

The results of the RCT are summarized in Table 1 [21-29]. Colin Ramirez et al. [21] assessed the effects of a nutritional intervention on the clinical and nutritional status and quality of life (QoL) in $65 \mathrm{HF}$ with reduced ejection fraction (HFrEF) and HF with preserved ejection fraction (HFpEF) patients assigned to an intervention group receiving a sodium-restricted diet (2,000 to 2,400 mg/day) with restriction of total fluids to $1.5 \mathrm{~L} /$ day or a control group that received traditional medical treatment and general nutritional recommendations. The intervention group had significantly less 


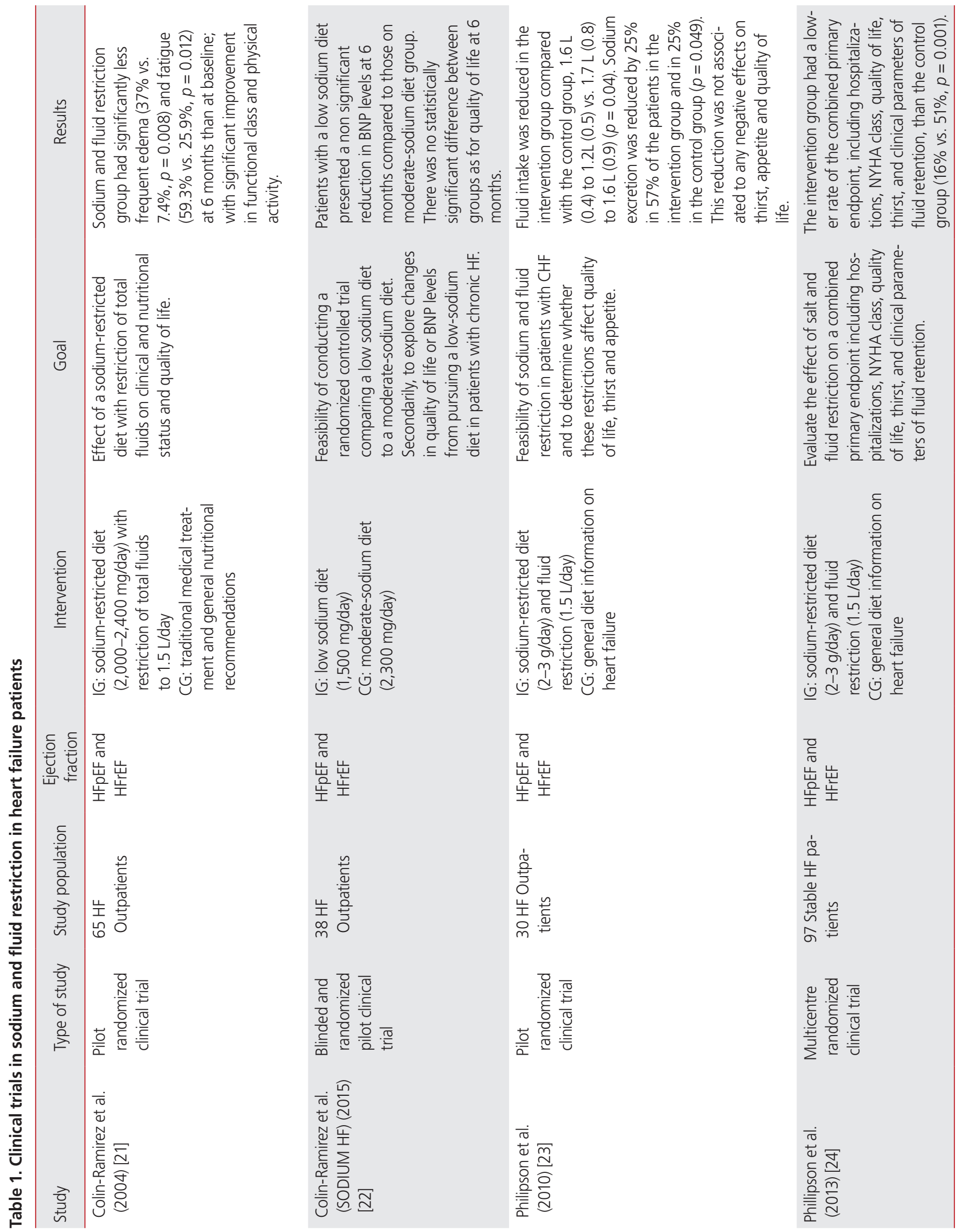




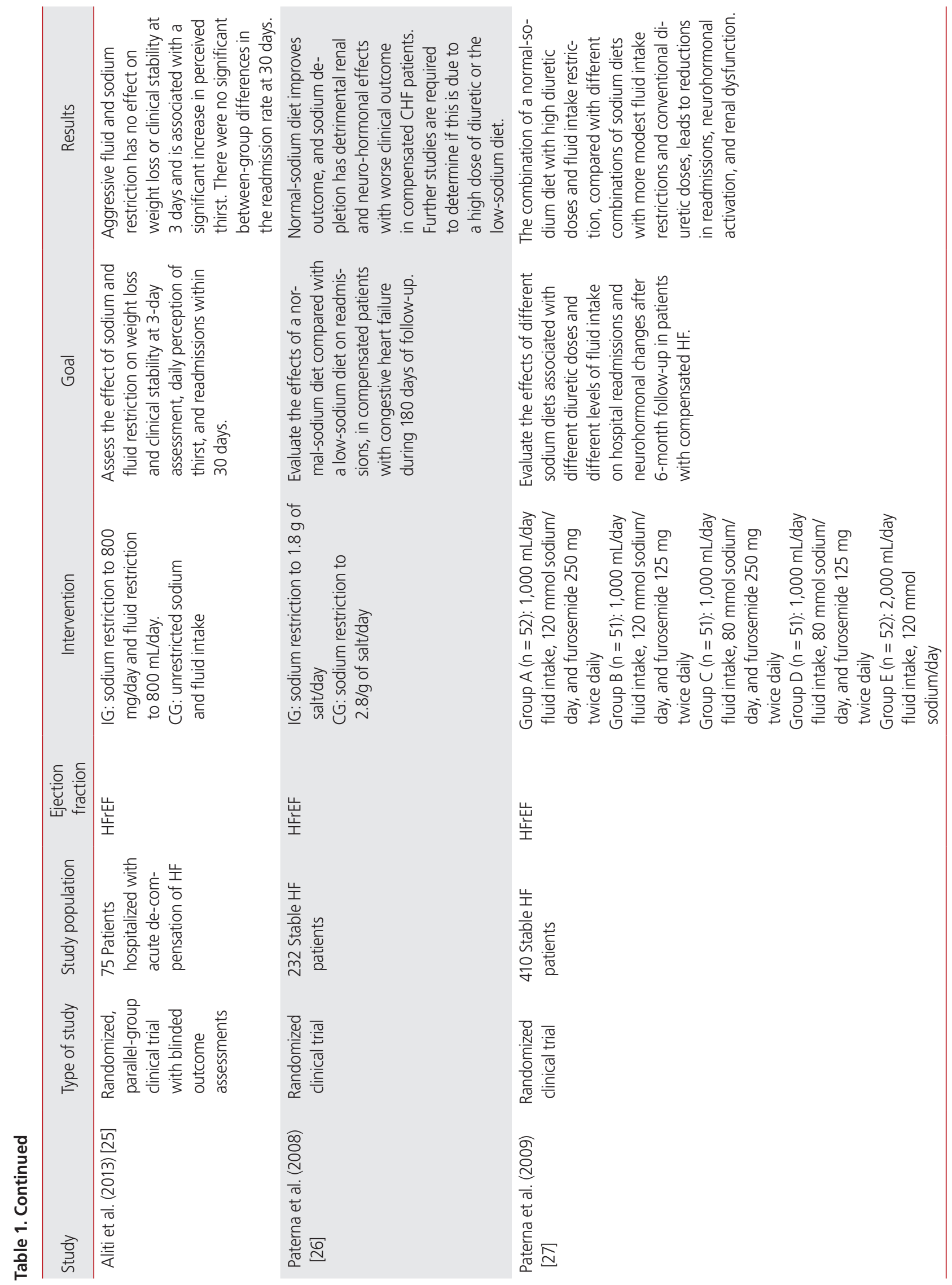




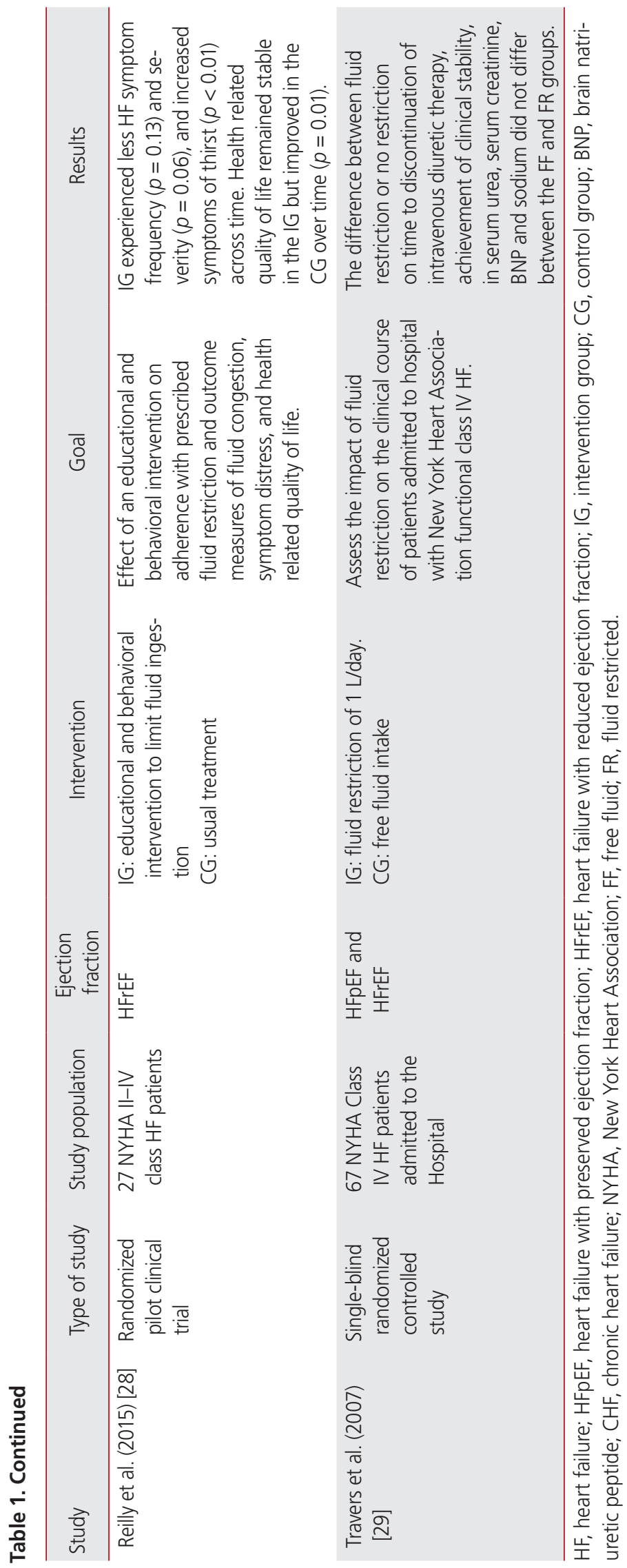

oedema (37\% vs. $7.4 \%, p=0.008)$ and fatigue $(59.3 \%$ vs. $25.9 \%, p=0.012$ ) at 6 months compared with baseline; in addition, functional class and physical activity improved significantly.

In a second, pilot RCT (the sodium HF), ColinRamirez et al. [22] compared low-sodium and moderate sodium diets in HFrEF and HFpEF patients. They included 38 patients, with HF New York Heart Association (NYHA) class II-III, who were randomized to 1.5 $\mathrm{g}$ of sodium daily or $2.3 \mathrm{~g} /$ daily. Patients on the low sodium diet showed a reduction in brain natriuretic peptide (BNP) levels at 6 months (mean change $51 \mathrm{pg} /$ $\mathrm{mL}, p=0.006$ ), while those on the moderate-sodium diet showed no changes in BNP, but the differences were not significant $(p=0.17)$. With respect to the QoL at 6 months, the median Kansas City Cardiomyopathy Questionnaire (KCCQ) clinical score increased from 63 to 75 in the low-sodium group and from 66 to 73 in the moderate-sodium group, with no significant differences ( $p=0.4$ between groups).

A pilot RCT by Philipson et al. [23] included 30 stable HFrEF and HFpEF patients who were randomized to an intervention group with information on how to reduce sodium intake to a maximum of 2 to $3 \mathrm{~g} /$ day and fluids to $1.5 \mathrm{~L} /$ day, or a control group who received usual care. The intervention significantly reduced sodium and fluid intake. Therefore, the same group [24] carried out a further RCT including 97 patients with stable HFrEF \& HFpEF (NYHA IIIV). Patients were randomized to sodium reduction to 2 to $3 \mathrm{~g} /$ day (measured as urinary sodium excretion), and fluid reduction to $1.5 \mathrm{~L}$ day, or to recommendations on general measures given by nurse-led HF clinics (do not drink too much and use salt with caution). The primary endpoint was a combination of NYHA class, hospitalization, weight, peripheral oedema, QoL, thirst, and need for diuretics. The intervention group had a lower rate of the combined primary endpoint, including hospitalizations, NYHA class, QoL, thirst, and clinical parameters of fluid retention than the control group ( $16 \%$ vs. $51 \%, p=0.001$ ).

Aliti et al. [25] randomized $75 \mathrm{HFrEF}$ patients to two groups, one with sodium restriction of $0.8 \mathrm{~g} / \mathrm{day}$ and fluid restriction of $800 \mathrm{~mL} /$ day versus an unrestricted group. Weight loss was similar in both groups. $(0.25 \mathrm{~kg}$; $95 \%$ confidence interval $[\mathrm{Cl}], 1.95$ to $2.45 ; p=0.82)$, as was the change in the clinical congestion score $(0.59$ points; $95 \% \mathrm{Cl}, 2.21$ to $1.03 ; p=0.47)$ at 3 days. Thirst 
was significantly worse in the intervention group $(5.1 \pm$ 2.9) than the control group $(3.44 \pm 2.0)$ and there were no significant differences in readmission rates at 30 days, (intervention group 11 patients, and control group seven patients, $p=0.41$ ).

Paterna et al. [26] analysed 6-month morbidity and mortality in 232 patients with $\mathrm{HF}$ randomized to $1.8 \mathrm{~g}$ of salt/ day or $2.8 \mathrm{~g} /$ day and found that patients with a stringent sodium reduction had a higher risk of mortality and hospital admission. The authors hypothesized that the results could be secondary to a high dose of diuretic or the low-sodium diet. Therefore, they [23] assessed the effects on hospital readmissions and neuro-hormonal changes, of two sodium diets, each with different diuretic doses and different levels of fluid intake, after 6 -months of follow-up in patients with compensated HFrEF. A total of 410 patients were randomized to eight groups, with different combinations of sodium and fluid restriction and different diuretic doses: group $A$ ( $n=52): 1,000 \mathrm{~mL}$ day fluid intake, $120 \mathrm{mmol}$ sodium/day, and furosemide $250 \mathrm{mg}$ twice daily; group $B(n=51): 1,000$ $\mathrm{mL} /$ day fluid intake, $2.76 \mathrm{~g}$ sodium/day, and furosemide $125 \mathrm{mg}$ twice daily; group C ( $\mathrm{n}=51): 1,000 \mathrm{~mL}$ day fluid intake, $1.84 \mathrm{~g}$ sodium/day, and furosemide $250 \mathrm{mg}$ twice daily; group $D(n=51): 1,000 \mathrm{~mL}$ day fluid intake, $1.84 \mathrm{~g}$ sodium/day, and furosemide $125 \mathrm{mg}$ twice daily; group $\mathrm{E}(\mathrm{n}=$ 52): 2,000 $\mathrm{mL}$ /day fluid intake, $2.76 \mathrm{~g}$ sodium/day. Patients receiving a combination of a normal-sodium diet, with high diuretic doses, and fluid intake restriction had a significantly greater reduction in readmissions, neuro-hormonal activation, and renal dysfunction compared with patients in the other groups.

With respect to fluid restriction, Reilly et al. [28] carried out a pilot RCT including 27 NYHA II-IV class HFrEF patients who were randomized to fluid restriction ( 1.5 to $2 \mathrm{~L} /$ day and education vs. usual clinical care). The results showed no differences in clinical measures of congestion. The group with fluid restriction reported fewer typical HF symptoms and less symptom severity but had greater thirst distress.

Travers et al. [29], in another single-blinded RCT including $67 \mathrm{HF}$ patients, compared the time to clinical stability between fluid restricted (FR; $n=34$ ) and free fluid (FF; $n=33$ ) groups, respectively. There were no significant differences $(8.3 \pm 6.3$ days vs. $7.0 \pm 6.0$ days, $p=0.17)$. There were no significant between-group differences in the time to discontinuation of intravenous diuretic therapy (FR: $2.7 \pm 4.5$ days, FF: $3.2 \pm 5.6$ days, $p=5.70$ ). The authors concluded that fluid restriction should be tested in a larger randomized controlled study.

\section{Observational studies and registries}

The results of these studies are summarized in Table 2 . In the GAP-HF study, Hummel et al. [30] made a pre-specified analysis, including 584 patients with HFrEF and 443 patients with HFpEF. They found that patients with HFpEF received less information on sodium restriction at hospital discharge than patients with HFrEF ( $42 \%$ vs. $53 \%, p=0.001)$. They also found that, in patients with $\mathrm{HFpEF}$, recommending significant sodium restriction was associated with a reduction in the combined variable of mortality and readmission within the first 30 days (odds ratio, $0.43 ; 95 \% \mathrm{Cl}, 0.24$ to 0.79 ; $p=0.007$ ).

Kollipara et al. [31] prospectively evaluated the correlation between gaps in knowledge about sodium content in the diet and the risk of readmission for HF in HFpEF and HFrEF patients. The rate of 90-day rehospitalizations for HF was 3-fold higher in patients with a low level of knowledge about sodium content than in the rest of the cohort $(28 \%$ vs. $9 \%, p=0.02$ ), and remained independently significant after adjustment for confounding factors.

A prospective observational study by Lennie et al. [32], which compared differences in event-free survival between patients with sodium intake above and below $3 \mathrm{~g}$ (stratified by NYHA functional class) included $302 \mathrm{HFrEF}$ and HFpEF patients. Sodium intake was estimated by 24-hour urinary sodium excretion due to the correlation with dietary sodium intake [33]. Patients in NYHA class III/IV with dietary sodium intake $>3 \mathrm{~g}$ were approximately 2.5-fold more likely to be hospitalized for heart problems or to die, after controlling for controlling for age, gender, aetiology of HF, BMI, left ventricular ejection fraction, and total comorbidity score. Conversely, sodium intake $<3 \mathrm{~g}$ was associated with a higher risk of hospitalization and death in patients in NYHA Class I/II, after adjusting for the same variables.

Song et al. [34] carried out a prospective study in $244 \mathrm{HF}$ patients that compared sodium excretion with outcomes. Patients were divided into three groups (intake $<2,2-3$, and $>3 \mathrm{~g} /$ day). Patients with a daily sodium intake $<2 \mathrm{~g} \mathrm{had}$ shorter event-free survival (hazard ratio [HR], 3.68; 95\% Cl, 1.18 to 11.50$)$, while patients with daily sodium intake $>3$ $\mathrm{g}$ had longer event-free survival $(\mathrm{HR}, 0.39 ; 95 \% \mathrm{Cl}, 0.16$ to 0.98) compared with those with an intake of 2 to $3 \mathrm{~g}$. Patients with a daily sodium intake $<2 \mathrm{~g}$ had a 3.7-fold higher 

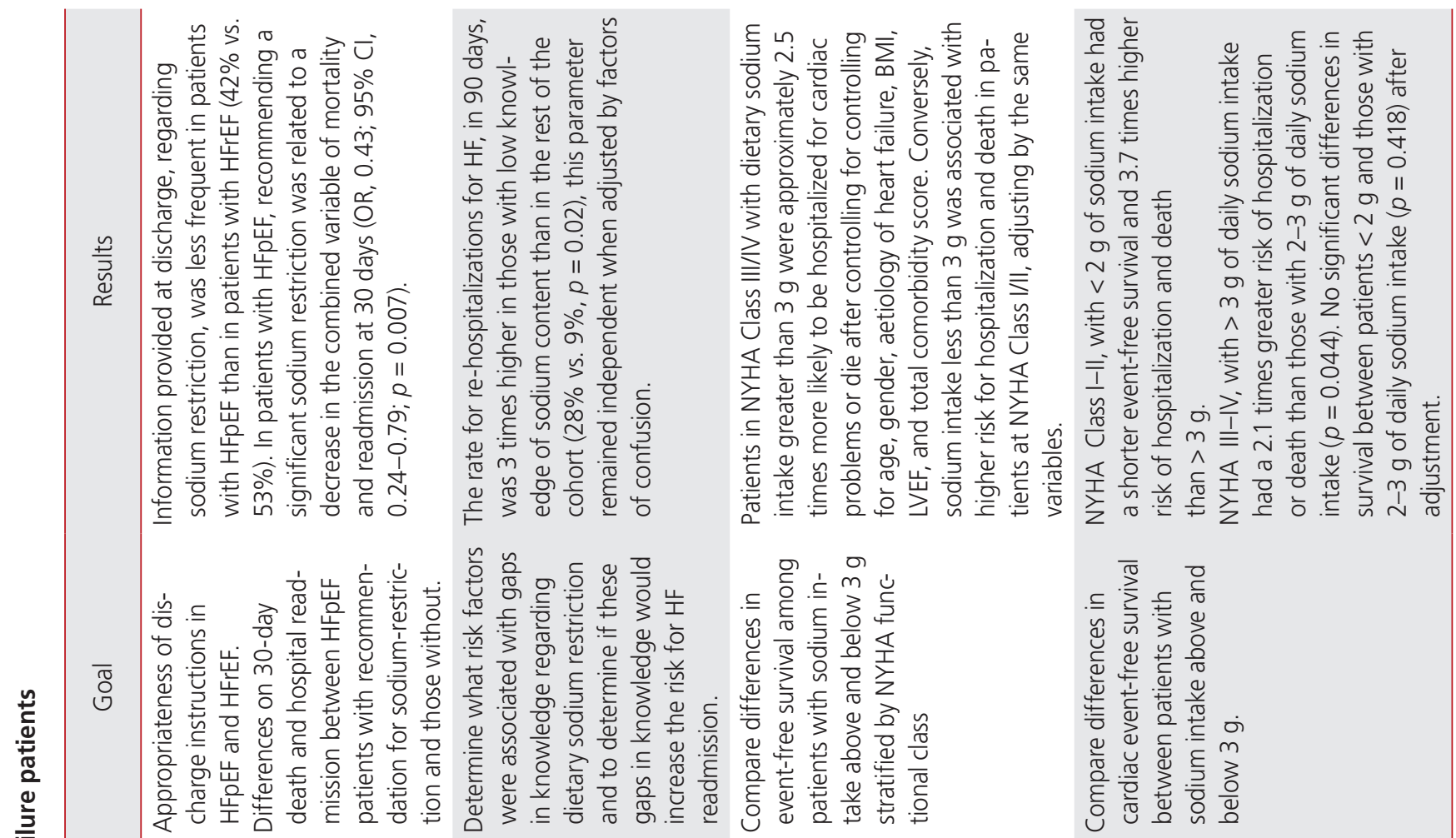

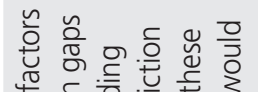

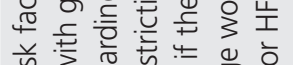

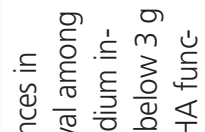

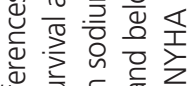

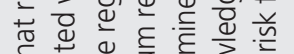

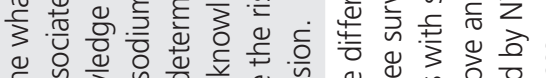

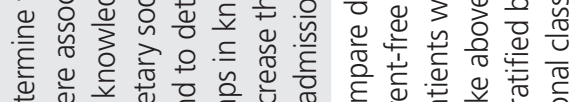

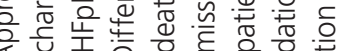

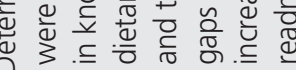

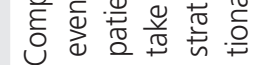
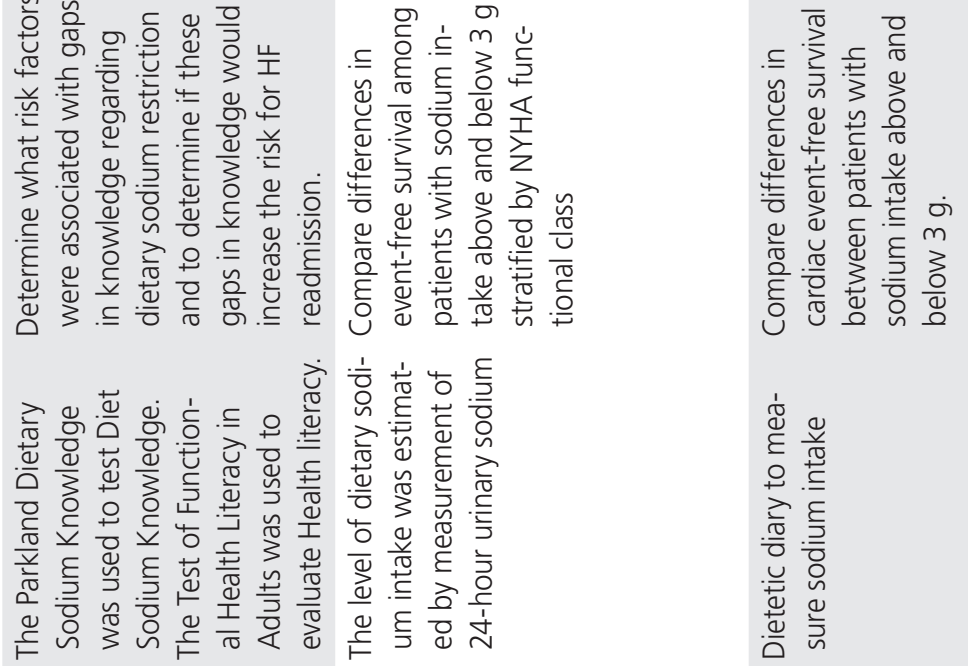

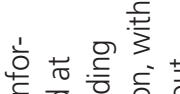

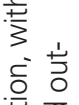

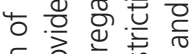
员言它岂

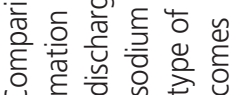

$\frac{2}{\frac{2}{3}}$

을

ह

든 을 을

通帝

岩岩㞱

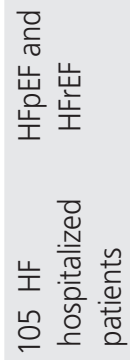

음
岩
岩崖
堊

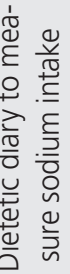

$\frac{1}{2}$

竞

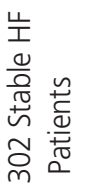

음

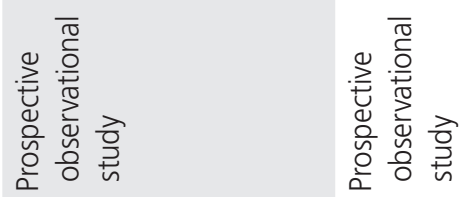

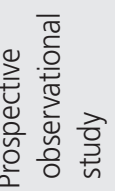
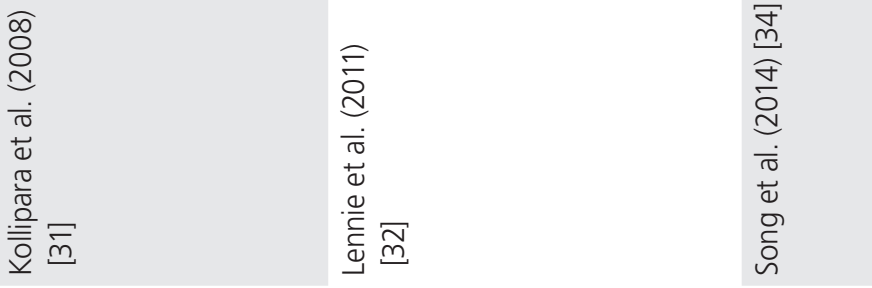


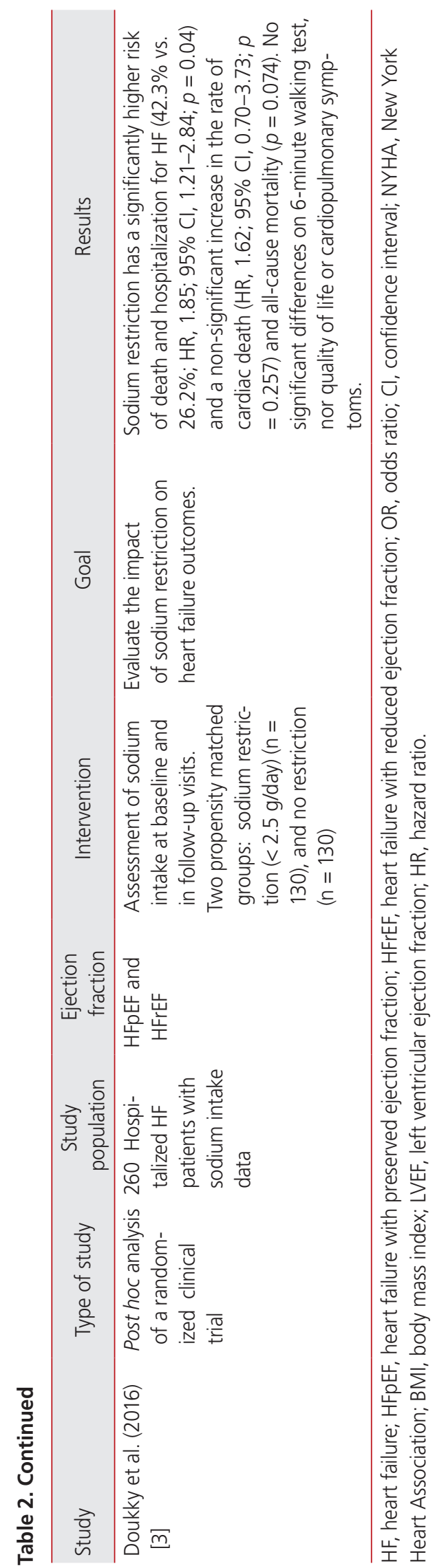

risk of hospitalization or death than those with an intake of 2 to $3 \mathrm{~g}(p=0.025)$. In contrast, patients with a daily sodium intake $>3 \mathrm{~g}$ had a $60 \%$ lower risk of hospitalization or death $(p=0.047)$. The results were consistent after adjustment for confounding factors. Patients with NYHA class III-IV and a daily sodium intake $>3 \mathrm{~g}$ had a 2.1-fold higher risk of hospitalization or death than those with an intake of 2 to $3 \mathrm{~g}(p=0.044)$. However, there were no significant differences in the adjusted survival curves between patients with $<2$ or $2-3 \mathrm{~g}$ of daily sodium intake $(p=0.418)$ after adjustment for the same covariates.

\section{Other studies}

The results of these studies are summarized in Table 2. A post hoc analysis of the multicentre randomized A Self-management Intervention for Mild to Moderate Heart Failure (HART) study by Doukky et al. [3] analysed data from 260 patients who were propensity matched to sodium restricted ( $n=130)$ and sodium unrestricted $(n=130)$ groups. They analysed the impact of sodium restriction in $\mathrm{HF}$ patients and concluded that sodium restriction ( $<2.5 \mathrm{~g} /$ day) was associated with a significantly higher risk of death and hospitalization for $\mathrm{HF}$ (42.3\% vs. 26.2\%; HR, 1.85; $95 \% \mathrm{Cl}, 1.21$ to $2.84 ; p=0.04)$ and a non-significant increase in the rate of cardiac death (HR, 1.62; $95 \% \mathrm{Cl}, 0.70$ to $3.73 ; p=$ 0.257 ) and all-cause mortality $(p=0.074)$. They also found no significant differences in the impact of sodium restriction on the 6-minute walking test, the QoL or cardiopulmonary symptoms.

\section{DISCUSSION}

This systematic review found a lack of clear evidence on the benefits of sodium/fluid restriction in chronic HF. The evidence is limited to a small number of studies with conflicting results. Large multicentre RCT targeting HF are lacking, and the available data come from pilot RCT, subgroup analyses, observational or retrospective studies. Therefore, current guideline recommendations are mainly based on expert opinion and further evidence is needed.

The prognosis of HF has improved due to new pharmacological treatments and devices that have been incorporated into clinical practice, leading to greater survival and functional improvements. Nevertheless, non-pharmacological 
measures remain an essential element of treatment.

Sodium restriction is a cornerstone of blood pressure (BP) control and volume reduction. As BP is one of the main risk factors for HF and modulates its outcome, it seems logical that sodium restriction could be beneficial in $\mathrm{HF}$, and it is recommended in the HF clinical practice guidelines [4].

A specific problem, as shown by the literature, is the difficulty in assessing the amount of sodium included in the diet. Some authors, such as Arcand et al. [35], postulate that food records and 24-hour urine collection are useful in measuring sodium intake in patients with cardiovascular disease and patients with $\mathrm{HF}$ not receiving diuretic treatment due to alterations in sodium excretion.

It seems clear that reducing sodium in the diet by introducing dietary models such as Dietary Approaches to Stop Hypertension (DASH) (diet for hypertension) has a favourable effect on BP control, which seems to play a key role in the pathogenesis of HFpEF, despite the expected increase in aldosterone, especially in elderly patients, which may contribute to increased neurohormonal activation, worsen renal function and increase hospitalizations in patients with HFpEF [36].

In contrast to accepted ideas, numerous studies have shown that severe sodium restriction in HF may be not as beneficial as originally supposed and may even be harmful. Arcand and Newton [37] suggested that evidence from large studies support a potential adverse effect of sodium restriction in patients with $\mathrm{HF}$, especially if they are under intensive diuretic treatment and water restriction.

Oria et al. [38] assessed whether dietary sodium restriction should be recommended in patients with HF. They postulated that, in patients with established cardiovascular disorders, including HF, a J-curve phenomenon is likely. They suggest that moderate sodium restriction to below high consumption levels are beneficial, but this does not apply to severe restrictions, which may carry risks. Therefore, they recommended that, until further studies are carried out, reductions in sodium consumption to $<2.3 \mathrm{~g}$ daily should not be recommended, since this may have unfavourable effects in HF patients. On the other hand, hyponatremia is one of the most frequent hydro-electrolytic disorders in patients with HF requiring hospitalization. Dietary sodium restriction can promote hyponatremia which, in itself, is an indicator of a poor prognosis, longer hospitalization, and increased hospitalization [39].

Chloride is the main extracellular anion that accompanies sodium and that comes from dietary sources. Chloride levels below $<100 \mathrm{mEq} / \mathrm{L}$ are known to be an independent predictor of all-cause mortality and cardiac mortality, independently of sodium, bicarbonate, and diuretic use. Therefore, very severe sodium restrictions, when attached to chloride in common salt, can lead to hypochloraemia, which could be harmful for patients with HF [40].

One problem is the prevalence of HF worldwide, including Asia. In this region, sodium intake is a big concern and cardiovascular death and hospitalization rates are higher than in other regions. Very little data from Asia is available. Hwang and Kim [41] analysed ninety-one Korean patients with HF and found a mean sodium intake of 3,982 mg/day, with men consuming a significantly higher amount than women (4.207 g vs. $3.523 \mathrm{~g}$ ). However, there is no data about sodium restriction in Asian patients in general, who may receive greater benefits than other populations.

The effects of sodium restriction in patients with HF should be clarified, since the importance of individual variations must be considered, including factors such as the cause of $\mathrm{HF}$, the chronicity of HF, functional class, kidney function, age, race, comorbidity, and symptoms. In addition, it is important to establish an effective method to monitor dietary sodium intake [42].

One of the main limitations of this analysis lies in the heterogeneity of the available studies, which are also incomplete and present a low degree of evidence. The different dietary patterns with respect to salt consumption in different countries and the absence of completely reliable measures for its quantification are also a limitation in most studies.

In conclusion, more studies of non-pharmacological measures in HF are needed. Methods to determine the nutritional status of these patients are required due to the significant impact on mortality from cachexia and malnutrition. Sodium restriction needs to be redefined as it may lead to increased mortality or readmission in some patients with HF.

\section{Conflict of interest}

No potential conflict of interest relevant to this article was reported.

\section{REFERENCES}

1. Choi HM, Shin MS. Angiotensin receptor-neprilysin inhibitor for the treatment of heart failure: a review of recent evidence. 
Korean J Intern Med 2020;35:498-513.

2. Choi HM, Park MS, Youn JC. Update on heart failure management and future directions. Korean J Intern Med 2019;34:1143.

3. Doukky R, Avery E, Mangla A, et al. Impact of dietary sodium restriction on heart failure outcomes. JACC Heart Fail 2016;4:24-35.

4. Ponikowski P, Voors AA, Anker SD, et al. 2016 ESC guidelines for the diagnosis and treatment of acute and chronic heart failure: the task force for the diagnosis and treatment of acute and chronic heart failure of the European Society of Cardiology (ESC) developed with the special contribution of the Heart Failure Association (HFA) of the ESC. Eur Heart J 2016;37:2129-2200.

5. Kim MN, Park SM. Heart failure with preserved ejection fraction: insights from recent clinical researches. Korean J Intern Med 2020;35:514-534.

6. Land MA, Neal BC, Johnson C, Nowson CA, Margerison C, Petersen KS. Salt consumption by Australian adults: a systematic review and meta-analysis. Med J Aust 2018;208:75-81.

7. Hipgrave DB, Chang S, Li X, Wu Y. Salt and sodium intake in China. JAMA 2016;315:703-705.

8. Kim YC, Koo HS, Kim S, Chin HJ. Estimation of daily salt intake through a 24-hour urine collection in Pohang, Korea. J Korean Med Sci 2014;29 Suppl 2:S87-S90.

9. Johnson C, Praveen D, Pope A, et al. Mean population salt consumption in India: a systematic review. J Hypertens 2017;35:3-9.

10. Kweon S, Kim Y, Jang MJ, et al. Data resource profile: the Korea National Health and Nutrition Examination Survey (KNHANES). Int J Epidemiol 2014;43:69-77.

11. Rhee MY, Shin SJ, Park SH, Kim SW. Sodium intake of a city population in Korea estimated by 24-h urine collection method. Eur J Clin Nutr 2013;67:875-880.

12. Dewan $P$, Jhund PS, Shen $L$, et al. Heart failure with reduced ejection fraction: comparison of patient characteristics and clinical outcomes within Asia and between Asia, Europe and the Americas. Eur J Heart Fail 2019;21:577-587.

13. Park JJ, Choi DJ. Current status of heart failure: global and Korea. Korean J Intern Med 2020;35:487-497.

14. Wong MM, Arcand J, Leung AA, Thout SR, Campbell NR, Webster J. The science of salt: a regularly updated systematic review of salt and health outcomes (December 2015-March 2016). J Clin Hypertens (Greenwich) 2017;19:322-332.

15. Estruch R, Ros E, Salas-Salvado J, et al. Primary prevention of cardiovascular disease with a Mediterranean diet sup- plemented with extra-virgin olive oil or nuts. N Engl J Med 2018;378:e34.

16. Williams B, Mancia G, Spiering W, et al. 2018 ESC/ESH guidelines for the management of arterial hypertension. Eur Heart J 2018;39:3021-3104.

17. Whelton PK, Carey RM, Aronow WS, et al. 2017 ACC/ AHA/AAPA/ABC/ACPM/AGS/APhA/ASH/ASPC/NMA/PCNA guideline for the prevention, detection, evaluation, and management of high blood pressure in adults: a report of the American College of Cardiology/American Heart Association Task Force on clinical practice guidelines. Hypertension 2018;71:e13-e115.

18. Lee HY, Shin J, Kim GH, et al. 2018 Korean Society of Hypertension guidelines for the management of hypertension: part II-diagnosis and treatment of hypertension. Clin Hypertens 2019;25:20.

19. Umemura S, Arima H, Arima S, et al. The Japanese Society of Hypertension guidelines for the management of hypertension (JSH 2019). Hypertens Res 2019;42:1235-1481.

20. Colin-Ramirez E, Arcand J, Ezekowitz JA. Estimates of dietary sodium consumption in patients with chronic heart failure. J Card Fail 2015;21:981-988.

21. Colin Ramirez E, Castillo Martinez L, Orea Tejeda A, Rebollar Gonzalez V, Narvaez David R, Asensio Lafuente E. Effects of a nutritional intervention on body composition, clinical status, and quality of life in patients with heart failure. Nutrition 2004;20:890-895.

22. Colin-Ramirez E, McAlister FA, Zheng Y, Sharma S, Armstrong PW, Ezekowitz JA. The long-term effects of dietary sodium restriction on clinical outcomes in patients with heart failure. The SODIUM-HF (Study of Dietary Intervention Under $100 \mathrm{mmol}$ in Heart Failure): a pilot study. Am Heart J 2015;169:274-281.

23. Philipson H, Ekman I, Swedberg K, Schaufelberger M. A pilot study of salt and water restriction in patients with chronic heart failure. Scand Cardiovasc J 2010;44:209-214.

24. Philipson H, Ekman I, Forslund HB, Swedberg K, Schaufelberger M. Salt and fluid restriction is effective in patients with chronic heart failure. Eur J Heart Fail 2013;15:1304-1310.

25. Aliti GB, Rabelo ER, Clausell N, Rohde LE, Biolo A, Beck-daSilva L. Aggressive fluid and sodium restriction in acute decompensated heart failure: a randomized clinical trial. JAMA Intern Med 2013;173:1058-1064.

26. Paterna S, Gaspare P, Fasullo S, Sarullo FM, Di Pasquale P. Normal-sodium diet compared with low-sodium diet in compensated congestive heart failure: is sodium an old enemy or 
a new friend? Clin Sci (Lond) 2008;114:221-230.

27. Paterna S, Parrinello G, Cannizzaro S, et al. Medium term effects of different dosage of diuretic, sodium, and fluid administration on neurohormonal and clinical outcome in patients with recently compensated heart failure. Am J Cardiol 2009;103:93-102.

28. Reilly CM, Higgins M, Smith A, Culler SD, Dunbar SB. Isolating the benefits of fluid restriction in patients with heart failure: a pilot study. Eur J Cardiovasc Nurs 2015;14:495-505.

29. Travers $B, O^{\prime}$ Loughlin $C$, Murphy NF, et al. Fluid restriction in the management of decompensated heart failure: no impact on time to clinical stability. J Card Fail 2007;13:128-132.

30. Hummel SL, DeFranco AC, Skorcz S, Montoye CK, Koelling TM. Recommendation of low-salt diet and short-term outcomes in heart failure with preserved systolic function. Am J Med 2009;122:1029-1036.

31. Kollipara UK, Jaffer $O$, Amin A, et al. Relation of lack of knowledge about dietary sodium to hospital readmission in patients with heart failure. Am J Cardiol 2008;102:12121215.

32. Lennie TA, Song EK, Wu JR, et al. Three gram sodium intake is associated with longer event-free survival only in patients with advanced heart failure. J Card Fail 2011;17:325-330.

33. McLean RM, Farmer VL, Nettleton A, et al. Twenty-four-hour diet recall and diet records compared with 24-hour urinary excretion to predict an individual's sodium consumption: a systematic review. J Clin Hypertens (Greenwich) 2018;20:13601376.

34. Song EK, Moser DK, Dunbar SB, Pressler SJ, Lennie TA. Dietary sodium restriction below $2 \mathrm{~g}$ per day predicted shorter event- free survival in patients with mild heart failure. Eur J Cardiovasc Nurs 2014;13:541-548.

35. Arcand J, Floras JS, Azevedo E, Mak S, Newton GE, Allard JP. Evaluation of 2 methods for sodium intake assessment in cardiac patients with and without heart failure: the confounding effect of loop diuretics. Am J Clin Nutr 2011;93:535-541.

36. Hummel SL, Seymour EM, Brook RD, et al. Low-sodium DASH diet improves diastolic function and ventricular-arterial coupling in hypertensive heart failure with preserved ejection fraction. Circ Heart Fail 2013;6:1165-1171.

37. Arcand J, Newton GE. Dietary sodium reduction in heart failure: a challenge to the Cochrane Review. Am J Hypertens 2012;25:19.

38. Oria M, Harrison M, Stallings VA. Dietary Reference Intakes for Sodium and Potassium. Washington (DC): National Academies Press, 2019.

39. DiNicolantonio JJ, Chatterjee S, O'Keefe JH. Dietary salt restriction in heart failure: where is the evidence? Prog Cardiovasc Dis 2016;58:401-406.

40. McCallum L, Jeemon P, Hastie CE, et al. Serum chloride is an independent predictor of mortality in hypertensive patients. Hypertension 2013;62:836-843.

41. Hwang SY, Kim J. An examination of the association of cognitive functioning, adherence to sodium restriction and $\mathrm{Na} / \mathrm{K}$ ratios in Korean heart failure patients. J Clin Nurs 2016;25:1766-1776.

42. Colin-Ramirez E, Ezekowitz JA. Salt in the diet in patients with heart failure: what to recommend. Curr Opin Cardiol 2016;31:196-203. 